\title{
The 3D organization of genome in the nucleus: from the nucleosome to the 4D nucleome
}

\author{
Guohong $\mathrm{Li}^{*}$ \\ National Laboratory of Bio-macromolecules, CAS Center for Excellence in Biomacromolecules, Institute of Biophysics, Chinese Academy of \\ Sciences, Beijing 100101, China
}

Received May 3, 2020; published online May 9, 2020

Citation: Li, G. (2020). The 3D organization of genome in the nucleus: from the nucleosome to the 4D nucleome. Sci China Life Sci 63, 791-794. https://doi. org/10.1007/s11427-020-1723-y

The genetic information, stored in the linear sequences of DNA, encodes all of the genes that a living organism uses to produce proteins and RNAs essential for various cellular functions. In eukaryotes, however, genomic DNA is hierarchically and efficiently packaged into multiple-level chromatin structures within the nucleus, including the nucleosome, $30 \mathrm{~nm}$ fibers, chromatin loops, topology associated domains (TADs), chromatin compartments, and chromosome territories (Dekker et al., 2017; Li and Zhu, 2015; Luger et al., 1997). This orderly organized spatial structure of chromatin and its dynamics play important roles in all DNA-related biological processes, such as gene transcription, DNA replication, damage and repair, which are all important to basic human health ( $\mathrm{Li}$ and $\mathrm{Zhu}, 2015)$. Not surprisingly, recent studies found that abnormal changes in this organization can cause various human diseases. Therefore, it is critical to understand: (i) how the genetic information is organized and stored in a micron-level nucleus; (ii) how this orderly folded spatial chromatin structure is unpackaged for replicating or reading the genomic information as needed. In this special topic "3D genome and nuclear architecture", we present two reviews that summarize recent advances in the techniques for 3D genomics research and major architectural proteins regulating 3D genome and nuclear architecture in mammalian cells ( $\mathrm{Li}$ et al., 2020; Zhang and Li, 2020), and two original research

\footnotetext{
*Corresponding author: liguohong@sun5.ibp.ac.cn
}

articles that predict the in vivo $30 \mathrm{~nm}$ chromatin fiber structure during interphase of human fibroblasts by developing a novel " $30-\mathrm{C}$ " model and study the biological function of CTCF (CCCTC-binding factor) in making choice of enhancers at Pcdh $\alpha$ locus (Ma et al., 2020; Wu et al., 2020).

The nucleosome is the fundamental repeating unit of chromatin, which is comprised of $\sim 146$ base pairs (bp) of DNA wrapped around a histone octamer with 2 copies of each core histone proteins (i.e., H2A, H2B, H3 and H4) in a left-handed manner. The structure of nucleosome core particle (NCP) has been resolved at high resolution by X-ray crystallography (Luger et al., 1997). Nucleosomes are connected by a segment of linker DNA to form a "beads-on-astring" nucleosomal array with a diameter of $11 \mathrm{~nm}$. In the presence of linker histones $\mathrm{H} 1 / \mathrm{H} 5$, nucleosomes can be further compacted into a $30-\mathrm{nm}$ chromatin fiber, which is regarded as the secondary structure of chromatin ( $\mathrm{Li}$ and Zhu, 2015). Despite considerable efforts over the past four decades, the structure and functional dynamics of $30-\mathrm{nm}$ fibers still remain unanswered and represent one of the most challenging questions in molecular biology. Two basic classes of structural models were proposed for the $30-\mathrm{nm}$ fiber, namely the one-start solenoid model and the two-start cross-linker model. Richmond and colleagues reported the crystal structure of tetranucleosome at $9 \AA$ resolution in the absence of linker histone, revealing a zigzag configuration with two stacks of two nucleosomes (Schalch et al., 2005). Recently, the three-dimensional (3D) cryo-EM structures of 
the reconstituted 30-nm chromatin fibers containing linker histone $\mathrm{H} 1.4$ has been determined at the resolution of $11 \AA$, revealing a left-handed double helical structure twisted by tetranucleosomal units with a two-start zig-zag conformation (Song et al., 2014).

Chromatin fibers are further configured into higher-order organizations in the nucleus, including chromatin loops, topologically associating domains (TADs), and chromatin compartments. During the past twenty years, 3C (chromosome conformation capture)-based genomic approaches for mapping chromatin interactions, such as $\mathrm{Hi}-\mathrm{C}$ and ChIAPET, are yielding genome-wide chromatin interaction maps at unprecedented resolution (Dekker et al., 2002; Fullwood et al., 2009; Lieberman-Aiden et al., 2009). In addition, livecell imaging and super-resolution microscopic approaches, combined with application of new ways (e.g. CRISPR-Cas9based systems) to visualize loci and sub-nuclear structures are beginning to provide detailed views of the $3 \mathrm{D}$ organization and dynamics of chromatin in living cells (Bintu et al., 2018; Cremer et al., 2017; Ma et al., 2018; Ma et al., 2016; Mateo et al., 2019). With the development and applications of these high throughput 3C-based techniques and superresolution microscopic methods, the three-dimensional (3D) genomics emerges as a new research field. Notably, the National Institutes of Health (NIH) has launched the 4DN (4D Nucleome) consortium projects in 2014 (Dekker et al., 2017). The 4DN projects combine cutting-edge 3D genomics technologies, single-cell sequencing, and high-resolution imaging to study how the 3D genome is formed, maintained and reorganized in diverse cell types and conditions, including in single cells, in space and time (the fourth dimension). By integrating multidisciplinary technologies, 4DN projects promote a transition from the study of onedimensional genome sequence to the study of four-dimensional genome spatial structure and temporal dynamics. The research has great potential to illuminate causal relationships between genome structures and functions, in both normal and disease states. In this special topic, two invited reviews summarize the most recent progresses in new $3 \mathrm{D}$ genomics research technologies, such as improvements of the traditional Hi-C and ChIA-PET methods, new approaches based on non-proximal-ligation strategies, and imaging-based methods improved over the past few years (Li et al., 2020; Zhang and Li, 2020). Especially, they reviewed the CRISPRbased methods for functional validations of 3D genomics, which could be the forthcoming directions.

As a central-level chromatin structure between nucleosome arrays and higher-order organizations, the $30-\mathrm{nm}$ fiber is the fundamental units of transcriptionally dormant chromatin. Thus understanding the structure and dynamics of chromatin fibers, that is, transitions between accessible form of nucleosomal arrays and highly compacted form of $30 \mathrm{~nm}$ chromatin fibers, is key to illuminate the regulatory functions of chromatin organization in different biological processes (Li and $\mathrm{Zhu}, 2015$ ). Structural studies in vitro revealed that the $30 \mathrm{~nm}$ fiber exhibits a left-handed two-start helical structure twisted by tetranucleosomal units with a zig-zag conformation (Schalch et al., 2005; Song et al., 2014). However, evidence for the existence of a $30 \mathrm{~nm}$ chromatin fiber in vivo has been elusive and controversial. Limited by the resolution of Hi-C data, even the most precise $\mathrm{Hi}-\mathrm{C}$ (kilobase resolution) cannot provide detailed information for the $30 \mathrm{~nm}$ fiber in vivo (Oluwadare et al., 2019). However, a few recent studies have mapped local chromatin folding at nucleosome resolution by micro-C genomics or imaging approaches, providing support for a tri- or tetra-nucleosome motif in yeast (Hsieh et al., 2015; Ohno et al., 2019) and twostart helical fibers in mammalian cells (Grigoryev et al., 2016; Hsieh et al., 2020; Krietenstein et al., 2020). Interestingly, in contrast to yeast, a distinctive pattern of nucleosomal folding was found in mouse cells. Consistent with prior cryo-EM results (Song et al., 2014), mammalian cells contain an extended two-start zig-zag chromatin fiber up to ten nucleosomes away, which may consist of at least 2-3 tetra-nucleosome stacks (Hsieh et al., 2020). In addition, RICC-seq (Risca et al., 2017) measuring fragment length distribution (FLD) of DNA strand breaks induced by ionizing radiation also supported the existance of $30-\mathrm{nm}$ fiber structures in vivo due to the special spatial organization of a two-start zig-zag chromatin (Rydberg et al., 1998). In this special topic, Yang and colleagues developed a novel "30-C" model combining $30-\mathrm{nm}$ chromatin structure models with Hi-C data, which measured the spatial contact frequency between different loci in the genome (Ma et al., 2020). Hi-C based chromatin interactions are used to infer spatial chromatin organization at larger scales, and $30-\mathrm{nm}$ fiber models are used to fill in nucleosome-level chromatin structures to achieve higher resolution. Finally, experimental data of ionizing radiation induced FLD and yields of single-strand break (SSB) and double-strand break (DSB) were used to validate the 30-C model, which predicts that the most probable chromatin fiber structure for human interphase fibroblasts in vivo is $45 \%$ zig-zag $30 \mathrm{~nm}$ fibers and $55 \% 10 \mathrm{~nm}$ fibers.

The human genome contains over 20,000 genes and a larger number of regulatory elements that are thought to be important for developmental gene regulation (ENCODE Project Consortium, 2012). In many cases, enhancers are key regulatory elements communicating with their target promoters through long-distance chromatin looping to orchestrate precise gene expression (Dekker and Mirny, 2016; Mifsud et al., 2015; Sanyal et al., 2012). In addition, one promoter may be regulated by multiple enhancers and one enhancer may regulate several promoters (Mifsud et al., 2015; Sanyal et al., 2012). These regulatory complexities offer a great challenge to pinpoint the specificity of long- 
distance promoter-enhancer interactions. CTCF, the CCCTC-binding factor that directionally binds to a vast range of genomic sites known as CBSs (CTCF-binding sites), mediates oriented chromatin looping between a substantial set of distal enhancers and target promoters (Guo et al., 2015). The chromatin looping mediated by CTCF often occurs between the forward-reverse convergent CBS pairs. This preferred configuration of CBS pairs is thought to underlie the formation of topologically associating domains (TADs) within which additional chromatin interactions regulate specificity of enhancer-promoter contacts (de Wit et al., 2015; Franke et al., 2016; Guo et al., 2015; Rao et al., 2014). In this special topic, Wu and colleagues used CRISPR/Cas9 based DNA-fragment editing to duplicate CBS-containing enhancers and promoters in the native genomic locus of the clustered Pcdh $\alpha$ genes to investigate mechanisms by which CTCF engages in enhancer choice (Wu et al., 2020). They found that the promoter is regulated by the proximal one among duplicated enhancers and that this choice is dependent on CTCF-mediated directional enhancer-promoter looping. These findings have important implications regarding the role of CTCF in specific interactions between enhancers and the target promoters as well as developmental regulation of gene expression by enhancer switching.

In addition to CTCF, the cohesion complex, many transcription factors and architectural proteins have been shown to be essential for 3D genome organization and controlling precise spatiotemporal gene expression patterns (Nora et al., 2017; Rao et al., 2017; Weintraub et al., 2017; Wutz et al., 2017). The architecture proteins, such as CTCF and YY1, organize the genome into different functional domains and determine enhancer-promoter communications (Guo et al., 2015; Weintraub et al., 2017). In this special topic, Li and colleagues introduce major architectural proteins that regulate $3 \mathrm{D}$ chromatin organization in mammalian cells, such as $\mathrm{CTCF}$, the cohesin complex, lamins, transcription factors and chromatin architectural proteins, and discuss relevant mechanisms such as phase separation in 3D genome organization ( $\mathrm{Li}$ et al., 2020). We hope these reviews and original research articles can provide insights that advance the future study of 3D genome and nuclear architecture, as well as other related fields.

Compliance and ethics The author(s) declare that they have no conflict of interest.

\section{References}

Bintu, B., Mateo, L.J., Su, J.H., Sinnott-Armstrong, N.A., Parker, M., Kinrot, S., Yamaya, K., Boettiger, A.N., and Zhuang, X. (2018). Superresolution chromatin tracing reveals domains and cooperative interactions in single cells. Science 362, pii: eaau1783.

Cremer, C., Szczurek, A., Schock, F., Gourram, A., and Birk, U. (2017). Super-resolution microscopy approaches to nuclear nanostructure imaging. Methods 123, 11-32. de Wit, E., Vos, E.S.M., Holwerda, S.J.B., Valdes-Quezada, C., Verstegen, M.J.A.M., Teunissen, H., Splinter, E., Wijchers, P.J., Krijger, P.H.L., and de Laat, W. (2015). CTCF binding polarity determines chromatin looping. Mol Cell 60, 676-684.

Dekker, J., Belmont, A.S., Guttman, M., Leshyk, V.O., Lis, J.T., Lomvardas, S., Mirny, L.A., O'Shea, C.C., Park, P.J., Ren, B., et al. (2017). The 4D nucleome project. Nature 549, 219-226.

Dekker, J., and Mirny, L. (2016). The 3D genome as moderator of chromosomal communication. Cell 164, 1110-1121.

Dekker, J., Rippe, K., Dekker, M., and Kleckner, N. (2002). Capturing chromosome conformation. Science 295, 1306-1311.

ENCODE Project Consortium (2012). An integrated encyclopedia of DNA elements in the human genome. Nature 489, 57-74.

Franke, M., Ibrahim, D.M., Andrey, G., Schwarzer, W., Heinrich, V., Schöpflin, R., Kraft, K., Kempfer, R., Jerković, I., Chan, W.L., et al. (2016). Formation of new chromatin domains determines pathogenicity of genomic duplications. Nature 538, 265-269.

Fullwood, M.J., Liu, M.H., Pan, Y.F., Liu, J., Xu, H., Mohamed, Y.B., Orlov, Y.L., Velkov, S., Ho, A., Mei, P.H., et al. (2009). An oestrogenreceptor- $\alpha$-bound human chromatin interactome. Nature 462, 58-64.

Grigoryev, S.A., Bascom, G., Buckwalter, J.M., Schubert, M.B., Woodcock, C.L., and Schlick, T. (2016). Hierarchical looping of zigzag nucleosome chains in metaphase chromosomes. Proc Natl Acad Sci USA 113, 1238-1243.

Guo, Y., Xu, Q., Canzio, D., Shou, J., Li, J., Gorkin, D.U., Jung, I., Wu, H., Zhai, Y., Tang, Y., et al. (2015). CRISPR inversion of CTCF sites alters genome topology and enhancer/promoter function. Cell 162, 900-910.

Hsieh, T.-H.S., Cattoglio, C., Slobodyanyuk, E., Hansen, A.S., Rando, O.J., Tjian, R., and Darzacq, X. (2020). Resolving the 3D landscape of transcription-linked mammalian chromatin folding. Mol Cell, pii: S1097-2765(20)30150-7.

Hsieh, T.H.S., Weiner, A., Lajoie, B., Dekker, J., Friedman, N., and Rando, O.J. (2015). Mapping nucleosome resolution chromosome folding in yeast by Micro-C. Cell 162, 108-119.

Krietenstein, N., Abraham, S., Venev, S.V., Abdennur, N., Gibcus, J., Hsieh, T.-H.S., Parsi, K.M., Yang, L., Maehr, R., Mirny, L.A., et al. (2020). Ultrastructural details of mammalian chromosome architecture. Mol Cell, pii: S1097-2765(20)30151-9.

Li, G., and Zhu, P. (2015). Structure and organization of chromatin fiber in the nucleus. FEBS Lett 589, 2893-2904.

Li, M., Gan, J., Sun, Y., Xu, Z., Yang, J., Sun, Y., and Li, C. (2020). Architectural proteins for the formation and maintenance of the $3 \mathrm{D}$ genome. Sci China Life Sci 63, 795-810.

Lieberman-Aiden, E., van Berkum, N.L., Williams, L., Imakaev, M., Ragoczy, T., Telling, A., Amit, I., Lajoie, B.R., Sabo, P.J., Dorschner, M.O., et al. (2009). Comprehensive mapping of long-range interactions reveals folding principles of the human genome. Science 326, 289-293.

Luger, K., Mäder, A.W., Richmond, R.K., Sargent, D.F., and Richmond, T. J. (1997). Crystal structure of the nucleosome core particle at $2.8 \AA$ resolution. Nature 389, 251-260.

Ma, H., Tu, L.C., Naseri, A., Chung, Y.C., Grunwald, D., Zhang, S., and Pederson, T. (2018). CRISPR-Sirius: RNA scaffolds for signal amplification in genome imaging. Nat Methods 15, 928-931.

Ma, H., Tu, L.C., Naseri, A., Huisman, M., Zhang, S., Grunwald, D., and Pederson, T. (2016). Multiplexed labeling of genomic loci with dCas9 and engineered sgRNAs using CRISPRainbow. Nat Biotechnol 34, $528-530$.

Ma, W., Gu, C., Ma, L., Fan, C., Zhang, C., Sun, Y., Li, C., and Yang, G. (2020). Mixed secondary chromatin structure revealed by modeling radiation-induced DNA fragment length distribution. Sci China Life Sci $63,825-834$.

Mateo, L.J., Murphy, S.E., Hafner, A., Cinquini, I.S., Walker, C.A., and Boettiger, A.N. (2019). Visualizing DNA folding and RNA in embryos at single-cell resolution. Nature 568, 49-54.

Mifsud, B., Tavares-Cadete, F., Young, A.N., Sugar, R., Schoenfelder, S., Ferreira, L., Wingett, S.W., Andrews, S., Grey, W., Ewels, P.A., et al. 
(2015). Mapping long-range promoter contacts in human cells with high-resolution capture Hi-C. Nat Genet 47, 598-606.

Nora, E.P., Goloborodko, A., Valton, A.L., Gibcus, J.H., Uebersohn, A., Abdennur, N., Dekker, J., Mirny, L.A., and Bruneau, B.G. (2017). Targeted degradation of CTCF decouples local insulation of chromosome domains from genomic compartmentalization. Cell 169, 930-944.e22.

Ohno, M., Ando, T., Priest, D.G., Kumar, V., Yoshida, Y., and Taniguchi, Y. (2019). Sub-nucleosomal genome structure reveals distinct nucleosome folding motifs. Cell 176, 520-534.e25.

Oluwadare, O., Highsmith, M., and Cheng, J. (2019). An overview of methods for reconstructing 3-D chromosome and genome structures from Hi-C data. Biol Proced Online 21: 7.

Rao, S.S.P., Huang, S.C., Glenn St Hilaire, B., Engreitz, J.M., Perez, E.M., Kieffer-Kwon, K.R., Sanborn, A.L., Johnstone, S.E., Bascom, G.D., Bochkov, I.D., et al. (2017). Cohesin loss eliminates all loop domains. Cell 171, 305-320.e24.

Rao, S.S.P., Huntley, M.H., Durand, N.C., Stamenova, E.K., Bochkov, I.D., Robinson, J.T., Sanborn, A.L., Machol, I., Omer, A.D., Lander, E.S., et al. (2014). A 3D map of the human genome at kilobase resolution reveals principles of chromatin looping. Cell 159, 1665-1680.

Risca, V.I., Denny, S.K., Straight, A.F., and Greenleaf, W.J. (2017). Variable chromatin structure revealed by in situ spatially correlated DNA cleavage mapping. Nature 541, 237-241.

Rydberg, B., Holley, W.R., Mian, I.S., and Chatterjee, A. (1998).
Chromatin conformation in living cells: support for a zig-zag model of the $30 \mathrm{~nm}$ chromatin fiber 1 1Edited by T. Richmond. J Mol Biol $284,71-84$.

Sanyal, A., Lajoie, B.R., Jain, G., and Dekker, J. (2012). The long-range interaction landscape of gene promoters. Nature 489, 109-113.

Schalch, T., Duda, S., Sargent, D.F., and Richmond, T.J. (2005). X-ray structure of a tetranucleosome and its implications for the chromatin fibre. Nature 436, 138-141.

Song, F., Chen, P., Sun, D., Wang, M., Dong, L., Liang, D., Xu, R.M., Zhu, P., and Li, G. (2014). Cryo-EM study of the chromatin fiber reveals a double helix twisted by tetranucleosomal units. Science 344, 376-380.

Weintraub, A.S., Li, C.H., Zamudio, A.V., Sigova, A.A., Hannett, N.M., Day, D.S., Abraham, B.J., Cohen, M.A., Nabet, B., Buckley, D.L., et al. (2017). YY1 is a structural regulator of enhancer-promoter loops. Cell 171, 1573-1588.e28.

Wu, Y., Jia, Z., Ge, X., and Wu, Q. (2020). Three-dimensional genome architectural CCCTC-binding factor makes choice in duplicated enhancers at $P c d h \alpha$ locus. Sci China Life Sci 63, 835-844.

Wutz, G., Várnai, C., Nagasaka, K., Cisneros, D.A., Stocsits, R.R., Tang, W., Schoenfelder, S., Jessberger, G., Muhar, M., Hossain, M.J., et al. (2017). Topologically associating domains and chromatin loops depend on cohesin and are regulated by CTCF, WAPL, and PDS5 proteins. EMBO J 36, 3573-3599.

Zhang, Y., and Li, G. (2020). Advances in technologies for 3D genomics research in recent years. Sci China Life Sci 63, 811-824.

\section{Biographical sketch}

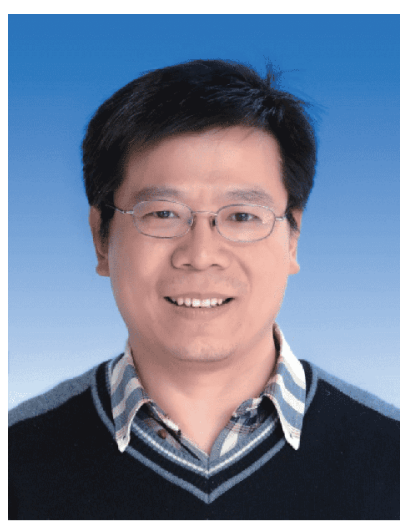

Dr. Guohong Li is currently an investigator of Institute of Biophysics of Chinese Academy of Sciences (Beijing, China). Dr. Li received his undergraduate degree from Wuhan University and his Master of Science from Peking University Health Center in 1998 and his Doctor of Philosophy (Dr. Rer. Nat.) degree from Max-Planck Institute for Cell Biology/Heidelberg University in 2003. Dr. Li joined the Institute of Biophysics, CAS (Beijing) in 2010 as a CAS Hundred Talents Investigator. Dr. Li's group has been mainly focusing on studying the biological functions of higher-order chromatin structures, especially the 30-nm chromatin fiber, in epigenetic regulation of gene expression, as well as the underlying molecular mechanisms. In the past years, Dr. Li's group has performed systematical research works and has made significant progresses on the structure and function of 30-nm chromatin fiber, the structure and function of centromeric chromatin, and epigenetic regulation of chromatin dynamics during development and in diseases. Dr. Li have published more than 60 research articles in peer-reviewed journals including Science, Nature, Nature Cell Biology, Molecular Cell, Developmental Cell, Genes \& Development, and Cell Research etc. Dr. Li serves on editorial boards of Science China Life Sciences, Genome Biology and the Journal of Biological Chemistry, and was selected as an HHMI International Research Scholar in 2017. 